\title{
Zum Verhältnis von Strafrecht und Maßregelrecht am Beispiel der Sicherungsverwahrung1
}

\section{Zur gegenwärtigen Situation der Sicherungsverwahrung in der Gesetzgebung und der kriminalpolitischen Diskussion}

Die Sicherungsverwahrung führte in Theorie und Praxis bis zum Gesetz zur Bekämpfung von Sexualstraftaten von 1998 und auch noch danach in Theorie und Praxis ein Schattendasein, bis sich der Gesetzgeber in diesem Jahrzehnt ihrer intensiv annahm und diese zu einem vieldiskutierten und zu einem von den Medien vereinnahmten Thema geworden ist, welches die Kriminalpolitik schon zeitweise zu dominieren schien.

Im Nachkriegsdeutschland wurde dieses Sicherungsmittel gegen gefährliche Straftäter als rechtsstaatlich bedenkliche Maßnahme über Jahrzehnte hinweg kritisch gesehen und viele Gerichte schreckten in der Praxis vor ihrer Anordnung zurück. ${ }^{2}$

Historisch geht die Sicherungsverwahrung ${ }^{3}$ auf das Gewohnheitsverbrechergesetz von 1933 zurück, ${ }^{4}$ ohne jedoch Ausdruck spezifisch nationalsozialistischen Rechtsdenkens gewesen zu sein, denn diese war bereits in den Strafrechtsreformentwürfen der 20er Jahre des vorigen Jahrhunderts vorgesehen gewesen und geht auf die sog. „,moderne" Strafrechtsschule Ende des 19. Jahrhunderts zurück. ${ }^{5}$ Im Rahmen der entnazifizierenden Strafrechtsbereinigungsgesetze von 1953 wurde diese in das StGB der Bundesrepublik übernommen. So auch die Regelung des $\S 2$ a RStGB. ${ }^{6}$ Dieser bestimmte zur zeitlichen Geltung, dass über Maßregeln der Besserung und Sicherung nach dem Gesetz zu entscheiden war, das zum Zeitpunkt der Entscheidung über die Maßregel galt. Erst in diesem Jahrzehnt erlangte diese Regelung praktische Bedeutung, als die zeitliche Ausweitung der Sicherungsverwahrung auf einen Altfall Anwendung fand.

Mit Inkrafttreten ${ }^{7}$ des Zweiten Gesetzes zur Reform des Strafrechts aus dem Jahr 1969, begrenzte der Gesetzgeber die Dauer der erstmalig angeordneten Sicherungsverwahrung auf zehn Jahre. Die vorgenannte Befristung hatte verschiedene Gründe: Die Scheu der Richter vor einer völlig unbestimmten Sanktion sollte beseitigt werden, das Verhältnismäßigkeitsprinzip und der Bestimmtheitsgrundsatz als Ausdruck rechtsstaat-

1 Der Aufsatz gibt den weitgehend unveränderten und um Anmerkungen ergänzten Text der Antrittsvorlesung wieder, welche der Verfasser am 15. Juli 2009 vor dem Fachbereich Rechtswissenschaft an der Johann Wolfgang Goethe-Universität im Rahmen seiner Umhabilitation gehalten hat.

2 Zur empirischen Realität der Unterbringung vgl. Böllinger, NK-StGB, 1. Aufl. 1995, § 66 Rn. 6 ff. m.w.N.

3 Zur Rechtsvergleichung siehe Böllinger, NK-StGB, 1. Aufl. 1995, § 61 Rn. 5 ff m.w.N.

4 Historischen Überblick geben Baumann/Weber, Strafrecht AT, 9. Aufl. 1985, S. 56, § 5 I 2 c; S. $710 \mathrm{f} ., \S 44 \mathrm{I} 1$.

5 Vgl. Baumann/Weber/Mitsch, Strafrecht AT, 11. Aufl. 2003, S. 67 f., § 6 Rn.18; Schmidhäuser, Strafrecht AT, 2. Aufl. 1975, S. 817, 21/4 m.w.N.

6 So $§ 2$ Abs. 4 StGB a. F., siehe Welzel, Das Deutsche Strafrecht, 11. Aufl. 1969, S. 26 m. w. N.; später seit dem 2. StrRG $\S 2$ Abs. 6 StGB.

7 Am 1.1.1975. 
licher Prinzipien wurden angeführt sowie weiterer Grund für die Befristung war die Unzulänglichkeit prognostischer Methoden hinsichtlich der Gefährlichkeit der in Frage kommenden Delinquenten.

Die Kriminalpolitik der sechziger und siebziger Jahre des vorigen Jahrhunderts war gekennzeichnet durch das Bestreben nach einer Zurückdrängung strafender staatlicher Reaktion. So konnte z. B. die Entkriminalisierung des Ladendiebstahls rechtspolitisch in Betracht gezogen und diskutiert werden. ${ }^{8}$ Die lebenslange Freiheitsstrafe geriet vor dem Bundesverfassungsgericht auf den Prüfstand. ${ }^{9}$ Nicht Strafrechtsverschärfung, sondern Strafrechtsbegrenzung war Anliegen der Reformen, die in diesen Jahrzehnten angestoßen wurden. Der Streit um den Schwangerschaftsabbruch und seine strafrechtliche Liberalisierung wurde bis weit hinein in die neunziger Jahre kontrovers ausgetragen. ${ }^{10}$

Die Sicherungsverwahrung war in dieser Zeit kein Thema. ${ }^{11}$ Aufgrund der vergleichsweise geringen Zahl von verwahrten Bürgern ${ }^{12}$ wurde sie in Theorie und Praxis nur wenig wahrgenommen. Politisch war aus ihr kein Kapital zu schlagen. Sofern zu dieser publiziert wurde, dann in kritischer bis ablehnender Distanz unter Einforderung rechtsstaatlicher Sicherungen gegen das Wegsperren. Reformer überlegten den Weg, wie die strafende Gesellschaft sich von der Verwahrung von Menschen verabschieden könne, ohne ihr Bedürfnis nach Sicherheit dadurch aufgeben zu müssen. Kriminalität wurde in diesen Jahrzehnten nicht als Bedrohung für den Durchschnittsbürger wahrgenommen. Politisch motivierte Kriminalität zog das öffentliche Interesse auf sich und forderte den Rechtsstaat heraus, so z.B. deutlich sichtbar anhand des bis heute in kraft gebliebenen Kontaktsperregesetzes. ${ }^{13}$ Zur Verteidigung des Rechtsstaates wurde seinerzeit eine Ausweitung des Anwendungsbereichs der Sicherungsverwahrung allerdings nicht ins Auge gefasst. ${ }^{14}$

Das Gesetz zur Bekämpfung von Sexualdelikten und anderen gefährlichen Straftaten von 1998 verschärfte im Gleichschritt mit dem zeitgleich verabschiedeten 6 . Strafrechtsreformgesetz ${ }^{15}$ erstmals die Sicherungsverwahrung, indem es zum einen die formellen Anordnungsvoraussetzungen senkte und zum anderen die zeitliche Obergrenze

8 Naucke, Gutachten D für den 51. Deutschen Juristentag, Bd. I Teil D/E, 1976.

9 BVerfGE 45, S. $187 \mathrm{ff}$.

10 Eine Vielzahl an Veröffentlichungen wurden hierzu geschrieben. Vgl. die Nachweise bei Eser in Schönke/Schröder, StGB, 27. Aufl. 2006, Vorbem. §§ $218 \mathrm{ff}$.

11 Näher Maurach/Gössel/Zipf, Strafrecht AT-Tb 2, 7.Aufl. 1989, S. 665, § 67 Rn. 3.

12 Siehe die Zahlen des Statistischen Bundesamtes bei Laubenthal, Strafvollzug, 5. Aufl. 2009, Rn. $869 \mathrm{f}$.

13 Nach $\S \S 31$ ff. EGGVG.

14 Vollzugsanstalten mit Hochsicherheitstrakten wie in Stammheim wurden geschaffen, um die Sicherheit des Strafvollzuges zu gewährleisten und die Außenkontakte vollständig überwachen zu können. Die weitere historische Entwicklung - zuletzt am Beispiel von Christian Klar - zeigte, dass kein Raum für eine Sicherungsverwahrung schon im Ansatz blieb auch aufgrund von fehlenden Gefährlichkeitsprognosen. Statistisch ist etwa die Hälfte der Sicherheitsverwahrten wegen Straftaten gegen die sexuelle Selbstbestimmung untergebracht.

$15 \mathrm{Zu}$ den zahlreichen Änderungen der Tatbestände des Besonderen Teils des StGB vgl. Dencker/Struensee/Nelles/Stein, Einführung in das 6. Strafrechtsreformgesetz 1998, München 1998. 
von 10 Jahren aufhob. ${ }^{16}$ Nach dem damals eingefügten Abs. 3 des $\S 66$ StGB kann das Gericht bereits nach der ersten Wiederholungstat die Sicherungsverwahrung anordnen und unter den Voraussetzungen des $\S 66$ Abs. 3 S. 2 StGB sogar ohne eine frühere Verurteilung oder Freiheitsentziehung. Das parallel verabschiedete 6. Strafrechtsreformgesetz beinhaltete insbesondere eine deutliche Erhöhung zahlreicher Strafrahmen und wurde deshalb als Strafverschärfungsgesetz zutreffend gekennzeichnet und kritisiert.

Diese geänderte rechtspolitische Einstellung fand ihre Fortsetzung in der Ausweitung des Anwendungsbereichs der Sicherungsverwahrung in den darauffolgenden Jahren. ${ }^{17}$ Entgegen den meist ablehnenden Stellungnahmen seitens der strafrechtlichen Berufe, verabschiedete die politische Mehrheit eine Reform nach der anderen bei der wiederoder neuentdeckten Sicherungsverwahrung als Mittel der Kriminalpolitik. Ungeachtet ihrer geringen praktischen Bedeutung rückte diese ins Zentrum der Aufmerksamkeit angesichts von einigen wenigen, von den Medien mit besonderer Aufmerksamkeit verfolgter Fälle gefährlicher und resozialisierungsresistenter Straftäter. ${ }^{18}$

Das statistische Zahlenmaterial der kriminologischen Forschung einschließlich der Rückfallzahlen stützte diese Renaissance der Sicherungsverwahrung in keinster Weise. ${ }^{19}$ Die empirischen Erhebungen zeigten vielmehr eine Entwicklung in die andere Richtung auf mit einem Rückgang von schweren Gewaltstraftaten und Rückfall nach langen Freiheitsstrafen oder Verwahrung. ${ }^{20}$

Gleichwohl vereinnahmte die Kriminalpolitik das Maßregelrecht als Objekt für Reformen mit letztendlich geringer praktischer Relevanz, womit allerdings ein breites juristisches Betätigungsfeld geschaffen war, mit welchem sich das Bundesverfassungsgericht wiederholt auseinandersetzen musste. Die hier gebundenen Energien ließen andererseits eine Kriminalpolitik vermissen, die sich der wirklichen Probleme im Bereich des Rechtsfolgenrechts angenommen hätte, wie beispielsweise der zu starken Belegung von Strafvollzugsanstalten.

Nicht Strafrechtsbegrenzung, sondern Ausweitung des Arsenals strafrechtlicher Reaktionsmöglichkeiten beherrschten das Credo populistischen strafrechtlichen Wirkens. Es sollte darum gehen, Gesetzeslücken zu schließen, welche nach Jahrzehnten gesetzgeberischer Untätigkeit im Maßregelrecht ausgemacht worden sein sollen. Die auf politische und juristische Klugheit gegründete Zurückhaltung im Maßregelrecht wurde weitestgehend aufgegeben und von der Verfassungsrechtssprechung abgesegnet.

$16 \mathrm{Zu}$ den Auswirkungen der Verschärfung auf den Straf- und Maßregelvollzug vgl. Bartsch/ Kreuzer, StV 2009, S. 54 ff.

17 Siehe im einzelnen Laubenthal, ZStW 116 (2004), S. 703 ff.

18 Kreuzer/Bartsch, GA 2008, S. 655 ff. m.w.N.

19 Siehe z. B. Böllinger, NK-StGB, 1. Aufl. 1995, §66 Rn. 32 a, welcher die symbolische Instrumentalisierung des Strafrechts für die scheinbare Bekämpfung gesellschaftlicher Risiken beklagt.

20 Kinzig, Die Legalbewährung gefährlicher Rückfalltäter. Zugleich ein Beitrag zur Entwicklung des Rechts der Sicherungsverwahrung, 2008. 


\section{Die Reaktionen in der Verfassungsrechtsprechung auf die Verschärfung der Sicherungsverwahrung}

Das Bundesverfassungsgericht hat in einer Entscheidung aus dem Jahr $2004^{21}$ den rückwirkenden Wegfall der Höchstfrist im seltenen Fall eines 47-jährigen, der seit seinem 15. Lebensjahr nur wenige Wochen in Freiheit verbracht hatte, für verfassungsgemäß erklärt, da es diesen Wegfall als im Einklang stehend mit dem rechtsstaatlichen Vertrauensgrundsatz angesehen hatte. ${ }^{22}$ Zugleich nahm das Verfassungsgericht indirekt Stellung zur Verfassungsmäßigkeit der Sicherungsverwahrung und bejahte diese. ${ }^{23}$ Es grenzte sie von der Strafe ab. Im Unterschied zur Verwahrung stelle die Strafe eine missbilligende hoheitliche Reaktion auf ein rechtswidriges, schuldhaftes Verhalten, ein Übel dar, das dem Schuldausgleich diene. Darauf sei der Anwendungsbereich des Art. 103 Abs. 2 GG als staatliche Maßnahme beschränkt, so dass er für die Sicherungsverwahrung nicht gelte, sondern bei ihr nur der allgemeine rechtsstaatliche Gesetzesvorbehalt und Bestimmtheits- bzw. Vertrauensgrundsatz. ${ }^{24}$

Zunächst wurde im Anschluss an das Gesetz zur Bekämpfung von Sexualstraftaten im Jahr 2002 die vorbehaltene Sicherungsverwahrung Gesetz in $\S 66$ a StGB. 2004 folgte die nachträgliche Anordnung der Unterbringung in $\S 66$ b StGB $^{25}$ und 2008 selbst die nachträgliche Sicherungsverwahrung nach Jugendstrafrecht in $\S 7$ Abs. $2-4$ JGG. ${ }^{26}$ Die Ausweitung der Anordnungs- und Prognosemöglichkeiten für die Unterbringung entsprach der kriminalpolitischen Stimmungslage seit Ende der 90er Jahre und nicht einem durch Fakten belegten Bedarf. ${ }^{27}$ Sie ist Abbild der kriminalpolitischen Wendung hin zum polizeilichen Präventionsstrafrecht. ${ }^{28}$ Die polizeiliche Kriminalstatistik für Jugendliche weist dagegen für das Jahr 2008 das Gegenteil mit einem Rückgang von $6 \%$ aus.

Die Entfesselung der Anordnungsmöglichkeiten für die Verwahrung von Delinquenten geht mit einem Wandel des Strafrechtsdenkens, der Konzeption von Strafrecht einher. Ein ,unreflektiert modernisiertes Bekämpfungs-Strafrecht“ ${ }^{29}$ mit einem kriminalpolitischen Konzept, das von Feindstrafrecht spricht und Polizeirecht in den Formen des

21 BVerfGE 109, S. $133 \mathrm{ff}$.

$22 \mathrm{Zu}$ Recht wird dieser Standpunkt kritisiert und eine Änderung des $\S 2$ Abs. 6 StGB eingefordert von Böllinger/Pollähne, NK-StGB, 2. Aufl. 2005, §61 Rn. 38. Zur Kritik an der Herausnahme der Maßregeln aus dem Anwendungsbereich von Art. 103 Abs. 2 GG als Ausdruck „unkritischen, affirmativen Naturrechts“, das u. a. Art. 103 Abs. 2 GG zu einer leblosen Vorschrift mache vgl. Naucke, W. Hassemer zum 60.Geburtstag, KritV 2000, S. 136. Vgl. Kinzig, NJW 2004, S. $911 \mathrm{ff}$.

23 Bereits in BVerfGE 2, S. 118 wurde die Verfassungswidrigkeit der Sicherungsverwahrung mit knapper Begründung verneint.

24 Schon nach früherer Rechtsprechung des BVerfG, NStZ-RR 1996, S. 122 (2 BvR 1734/90) verstoße die Anordnung der SV nicht gegen das Grundgesetz, insbesondere nicht gegen das Gebot der Gesetzesbestimmtheit und das Verbot der doppelten Bestrafung.

$25 \mathrm{Zu}$ den gesetzlichen Neuregelungen im einzelnen Passek, GA 2005, S. $96 \mathrm{ff}$.

26 Einzelheiten bei Streng, Jugendstrafrecht, 2. Aufl. 2008, Rn. 555 ff., $563 \mathrm{ff}$.

27 Fischer, StGB, 56. Aufl. 2009, § 66 b Rn. 4; Laubenthal, ZStW 116 (2004), S. 703 ff.

28 Dazu eingehend Hassemer, Strafrecht. Sein Selbstverständnis, seine Welt, 2008, S. 239 ff., $245 \mathrm{f}$. Zu weiteren aktuellen Reformvorhaben bei der SV vgl. Rissing van Scan/Peglau, LKStGB, 12. Aufl., § 66 b Rn. 16.

29 Fischer, StGB, a.a.O., Einl. Rn. 12 a. 
Strafrechts vollzieht ${ }^{30}$ ist Wegbereiter der beschriebenen Wiederbelebung der Sicherungsverwahrung und auch der Freiheitsstrafe. Diese kann sich auf einen realen praktischen Bedarf kaum beziehen und gesteht auch offen ein, dass der Anwendungsbereich beispielweise der nachträglichen Sicherungsverwahrung auf absolute Ausnahmefälle beschränkt sei. Die sog. Reformen zur Sicherungsverwahrung sind erneut Beispiel einer Vorratsgesetzgebung mit symbolischem Gehalt, ${ }^{31}$ aber einer Symbolik fragwürdigen, übertriebenen Gehalts und einem Sicherheitsdenken, ${ }^{32}$ dem alles Andere - insbesondere die Freiheit - untergeordnet wird. ${ }^{33}$

Strafe und Verwahrung jeweils aus Anlass einer tatbestandsmäßigen und rechtswidrigen Tat unterscheiden sich aufgrund ihrer Koppelung bzw. Lösung von der Schuld des Delinquenten. Die schuldunabhängig verhängten und vollstreckten Maßregeln, die im Fall der Sicherungsverwahrung auch das (- falls vorhandene -) Verschulden zeitlich weit überschreiten können, sind dadurch einem besonderen Legitimationsproblem ${ }^{34}$ ausgesetzt, das sich anhand rechtlicher Maßstäbe kaum lösen lässt. Daher wird auch von einem zweispurigen Rechtsfolgensystem gesprochen, das unterschiedlichen juristischen Klassifikationen folgt. Mit den Formeln der Strafe als Repression für begangenes Unrecht und den Maßregeln als Prävention vor künftigem Unrecht kann man diese verschiedenen Reaktionsformen vereinfachend gleichsetzen. Warum das zweispurige System unterschiedlichen juristischen Maßstäben folgt, ist damit allerdings nicht wirklich begründet worden.

Praktisch bedeutsam wurde dies bereits an dem erwähnten Beispiel einer echten Rückwirkung der nachträglichen Sicherungsverwahrung auf einen Fall, der bereits vor ihrer Anordnung mit Strafurteil abgeurteilt worden war, ohne dass die nachträgliche Anordnung der Sicherungsverwahrung zum Tatzeitpunkt im Gesetz vorgesehen war. Das BVerfG wendete das Rückwirkungsverbot des Art. 103 Abs. 2 GG nicht auf die Maßregel an und grenzte in seiner Entscheidung die repressive Strafe als Ausgleich für eine schuldhaft begangene Tat von dem mit der Sicherungsverwahrung verbundenen Sicherheitsinteresse der Allgemeinheit im präventiv-polizeirechtlichen Sinne ab.

Diese systemischen Abgrenzungen wurden schon vor Jahrzehnten als Etikettenschwindel ${ }^{35}$ bezeichnet und die Kritik formuliert, dass auf die Sicherungsverwahrung als in erster Linie freiheitsentziehender Maßnahme mit fraglichem resozialisierenden Gehalt nicht die gebotenen rechtsstaatlichen Garantien Anwendung finden würden. In der Tat stellt das BVerfG wie auch die überwiegend seine Auffassung stützende Meinung im Schrifttum auf diese funktionelle Verschiedenheit dieser beiden Instrumente

30 Fischer, a.a.O., § 261 Rn. 4 b.

31 Hierzu grundlegend Hassemer, NStZ 1989, S. 553-559 = ders., Strafen im Rechtsstaat, 2000, S. 170 ff. m.w.N.; ders., Festschrift für Roxin, 2001, S. 1001-1019 = ders., Strafrecht, 2008, S. 93 ff.; kritische Zusammenfassung dieser Tendenz bei P.-A. Albrecht, Kriminologie, 3. Aufl. 2005, S. 64 ff., 132.

32 Zum „nach-präventiven Sicherheitsstrafrecht“ vgl. die zusammenfassende Darstellungen bei P.-A. Albrecht, a.a.O., S. $72 \mathrm{ff} ., 132 \mathrm{ff}$.

33 Zur juristischen Kunstfigur eines „Grundrechts auf Sicherheit“ und Kritik an derselben vgl. P.-A. Albrecht, a.a.O., S. $101 \mathrm{f}$.

34 Vgl. zu einem ,besonderen Legitimationsdruck“ bei der SV Laubenthal, a.a.O.

35 Kohlrausch, ZStW 44, S. 21; Hall, ZStW 70 (1958), S. 59; H. Mayer, Strafrecht AT, 1967, S. 184, § 49 II 2; Jescheck/Weigend, a.a.O., S. 87, § 9 II 2. Vgl. dazu Böllinger, NK-StGB, 1. Aufl. 1995, § 61 Rn. 37 m.w.N.; Baumann/Weber, a.a.O., S. 711, § 44 I 1. 
aus der Sicht der Gesellschaft ab, um einmal die besonderen rechtsstaatlichen Garantien bei der Strafe Anwendung finden zu lassen und zum anderen lediglich die allgemeinen rechtsstaatlichen Standards, wie sie im Polizeirecht oder der Abschiebehaft gelten.

Diese Differenzierung beschäftigte das $\mathrm{BVerfG}^{36}$ erneut aus einem anderen Blickwinkel, als es um die Frage der Verteilung der Gesetzgebungskompetenz zwischen dem Bund und den Ländern ging anlässlich der Normierung der nachträglichen Sicherungsverwahrung durch einzelne Landesgesetzgeber. Die Grenzziehung zwischen Strafrecht und Polizeirecht fiel hier anders aus.

Das BVerfG entschied zugunsten des Bundes, dass diesem nach Art. 74 Abs. 1 Nr. 1 GG die Rechtsetzungsprärogative bei der Sicherungsverwahrung - auch der vorbehaltenen und nachträglichen - als Strafrecht zustehe, von welcher er bereits mittels von $\S 66$ StGB Gebrauch gemacht habe, so dass für eine konkurrierende Gesetzgebung der Länder kein Raum mehr sei. Die gesetzliche Regelung der nachträglichen Sicherungsverwahrung durch einzelne Bundesländer ${ }^{37}$ war somit nicht verfassungsgemäß, da diese nach Interpretation des BVerfG hiermit Strafrecht und nicht wie die Länder meinten, Polizeirecht normiert hätten.

Den Begriff des Strafrechts definierte das BVerfG hier nicht im Sinne des Art. 103 Abs. 2 GG anhand seiner repressiven Funktion gegenüber schuldhaft begangenem Unrecht, sondern anhand des Sachzusammenhangs der Regelungsmaterie Sicherungsverwahrung mit dem Strafrecht. Der Begriff des Strafrechts wird demnach vom BVerfG verschieden inhaltlich festgelegt anhand seiner Funktion, wenn es zum einen um die Reichweite und den Anwendungsbereich des Art. 103 Abs. 2 GG und seiner strafverfassungsrechtlichen Garantien geht und zum anderen, wenn die Frage der Verteilung der Gesetzgebungszuständigkeit zwischen dem Bund und den Ländern zu beantworten ist. Dies ist Ergebnis der folgenorientierten Interpretation des BVerfG. ${ }^{38}$

\section{Rechtsstaatliche Nichtbegrenzung der Sicherungsverwahrung}

Der Aufwand strafjuristischen Arbeitens ist ganz erheblich, um zu einer angemessenen Begrenzung des Umfangs von Strafbarkeit und Strafe zu gelangen. Was jedoch für die eine Spur des Strafrechtssystems Geltung beanspruchen kann, soll für die andere Spur der Maßregel der Sicherungsverwahrung, welche persönlich von den Betroffenen ganz ähnlich wie Strafe wahrgenommen und erlebt wird, kein Maßstab sein.

Es stimmt nicht nur nachdenklich, sondern gibt Anlass zum juristischen Zweifel, wenn einmal dem Strafjuristen hoher Aufwand in seiner juristischen Argumentation abverlangt wird, damit das staatliche Strafen rechtmäßig ist, nicht ausufert oder gar totalitäre Züge annimmt und dann zum anderen auf der zweiten Spur des Rechtsfolgenrechts vieles von dem wieder machbar wird, was zuvor beim ob und wie der Strafe aufgrund juristischer Vernunft nicht zulässig und möglich gewesen ist.

36 BVerfGE 109, S. $190 \mathrm{ff}$.

37 Vgl. Laubenthal, a.a.O., Rn. 876.

38 Vgl. hierzu die Grundlagen bei Hassemer, Festschrift für Coing, 1982, S. 493-524. 
Schon seit Langem wird dies vehement kritisiert und teils für verfassungswidrig gehalten und Ausschau nach Alternativen im Sozialrecht ${ }^{39}$ gehalten. So nahmen sich Gegner der Sicherungsverwahrung und Vielstraferei unter anderem der Sicherungsverwahrung an und forderten grundlegende Reformen des Rechtsfolgenrechts ein ${ }^{40}$ mit einem ganz anderen Vorverständnis als dieses gegenwärtig aufgrund des kriminalpolitischen Stimmungsumschwunges seit einem guten Jahrzehnt vorzufinden ist mit einer beständigen Verschiebung des Kräfteverhältnisses zwischen Bürger und Staat zugunsten kollektiver Interessen.

Gegenwärtig besteht weit weniger Aussicht, die Sicherungsverwahrung zurückzudrängen bzw. weitgehend ersetzen zu können als in den Jahrzehnten zuvor. Angesichts dieser Situation einer zu erwartenden, bleibenden Präsens der Sicherungsverwahrung und ihrer Ausweitung im System der Rechtsfolgen der Tat ist ihre Begrenzung und Zurückdrängung nötiger als zuvor.

Wie schwierig es ist, zu einer Begrenzung der Anwendungsmöglichkeiten der Sicherungsverwahrung zu gelangen, soll verdeutlicht werden. Es ist der Versuch, die Verwerfungen strafrechtlicher Prinzipien zwischen den Strafen und der Maßregel Sicherungsverwahrung aufzuzeigen und ist zugleich ein Beispiel dafür, dass eine Begrenzung der Sicherungsverwahrung mittels der vorhandenen Instrumentarien kaum zu leisten ist. Dieser Versuch macht zugleich deutlich, woher das schlechte Gewissen vieler Strafjuristen beim Blick auf die Sicherungsverwahrung rührt und die frühere Zurückhaltung vieler Richter, ${ }^{41}$ diese im Einzelfall anzuordnen.

Nicht auf die Fallgruppe der schuldunfähigen Straftäter soll im nachfolgenden die Aufmerksamkeit gerichtet werden, bei der eine Freiheitsstrafe wegen Schuldunfähigkeit ohnehin nicht in Betracht kommt. Das Interesse soll vielmehr denjenigen Fällen gelten, in denen die Sicherungsverwahrung als Maßregel neben der Strafe ergänzend angeordnet wird. Hier stellt sich abweichend von den Maßregeln der Unterbringung in einem psychiatrischen Krankenhaus oder in einer Entziehungsanstalt die Frage des Vorliegens einer Mehrfachsanktionierung mit besonderer Schärfe.

Die zuletzt genannten Maßregeln der behandelnden Unterbringung haben wenigstens ein Anrechnungssystem für die - in der Regel vorweg zu vollziehende - Maßregel auf die Strafe, so dass die Zeit im Strafvollzug durch den Maßregelvollzug verkürzt wird. Bei der Sicherungsverwahrung wird dagegen die Freiheitsstrafe grundsätzlich zuerst vollstreckt. Anschließend erfolgt die Unterbringung in der Sicherungsverwahrung.

Damit stellt die Maßregel neben der Freiheitsstrafe für eine schuldhaft begangene Tat eine zweite Sanktion dar, die gegen das Verbot der Doppelbestrafung des Art. 103 Abs. 3 GG verstößt. Die Strafjuristen überwinden dieses strafverfassungsrechtliche Problem mit Hilfe des Denkens und Argumentierens in dem beschriebenen zweispurigen Sanktionensystem.

Die Sicht- und Interpretierweise von Strafe und Maßregel mittels dieses Trennungsdenkens unter Hinweis auf die unterschiedlichen Zwecksetzungen der beiden Sanktionsformen löst jedoch das angesprochene strafverfassungsrechtliche Problem in keiner

39 So bereits H. Mayer, Strafrechtsreform für heute und morgen, 1962, S. 153 ff., 165; ders., Strafrecht AT, 1967, S. 179 f., 185; Naucke, Strafrecht, 10. Aufl. 2002, S. 99, 109, § 3 Rn. 33, 64.

40 Siehe m.w.N. Böllinger, NK-StGB, 1. Aufl. 1995, § 66 Rn. 41.

41 Maurach/Gössel/Zipf, a.a.O., S. 678, §68 Rn. 21. 
Weise. Im Unterschied zur Anordnungsstufe ist auf der Vollzugsstufe dieses zweispurige Sanktionensystem auch weitgehend durch ein einspuriges ersetzt worden, wenn auch das BVerfG mit seinem „Abstandsgebot" ${ }^{\star 42}$ eine inhaltliche Differenz von Freiheitsstrafe ${ }^{43}$ und Verwahrung im Sinne eines privilegierten Vollzuges einfordert. Besondere Justizvollzugsanstalten für die Unterbringung von Sicherungsverwahrten existieren in der Bundesrepublik Deutschland nicht, werden aber neuerdings als länderübergreifende neue Projekte angestrebt. In ihren Wirkungen und im praktischen Vollzug unterscheidet sich die Sicherungsverwahrung so gut wie nicht von der Freiheitsstrafe. ${ }^{44}$ Den Verwahrten werden lediglich ein paar Vergünstigungen eingeräumt im Vergleich zu Strafgefangenen, aber auch nur soweit diese nicht den praktischen Betriebsablauf der Anstalten stören.

Die Erörterung der Doppelbelastung von Straftätern durch die Sicherungsverwahrung wird in der strafrechtlichen Literatur oft vermieden ${ }^{45}$ oder aber vehement kritisiert. ${ }^{46}$ Unzutreffend wird von der überwiegenden Meinung die Notwendigkeit einer Sanktionenbegrenzung bei dieser aufgrund des Verfassungsgrundsatzes ne bis in idem wohl auch nicht anerkannt. ${ }^{47}$ In einschlägigen Kommentaren zum Grundgesetz wird dieses Problem kaum abgehandelt, eher abgetan. ${ }^{48}$

Die moderne Verfassungsdogmatik und das BVerfG neigen dazu, hier nicht Art. 103 Abs. 3 GG als Justizgrundrecht zugunsten von straffälligen Bürgern, sondern nur den allgemeinen rechtsstaatlichen Verhältnismäßigkeitsgrundsatz - gesetzlich konkretisiert in $\S 62 \mathrm{StGB}$ - anzuwenden ${ }^{49}$ bzw. Art. 103 Abs. 3 GG in seinem Sinne zu interpretieren.

42 BVerfGE 109, S. 133 ff. Vgl. im Einzelnen hierzu Laubenthal, ZStW 116 (2004), S. 730 ff. mit berechtigter Kritik aufgrund der tatsächlichen Vollzugsverhältnisse. Zur früheren Situation des Vollzuges der SV vgl. Hall, ZStW 70 (1958), S. 49 ff.

43 Vgl. näher zum privilegierten Vollzug Laubenthal, a.a.O., Rn. 874 b.

44 Siehe Laubenthal, a.a.O., Rn. 874; ders., ZStW 116 (2004), S. 710; Böllinger, NK-StGB, 1. Aufl. 1995, § 66 Rn.11. § 140 Abs. 1 StVollzG schreibt die getrennte Unterbringung der Sicherungsverwahrten von Strafgefangenen vor.

45 So schon die Bestandsaufnahme von Schmidhäuser, a.a.O., S. 819 Fn. 4, 21/8.

46 Köhler, a.a.O., S. 57 f., Kap.1/3.3 m.w.N., S. 642 f., Kap. 10/3.3.4., der ihre Aufhebung und Transformation in gerechte Strafe fordert, welche sich ,,auf habituelle Delinquenz nach Schwere und Bewährungsregelung maßgerecht bezieht".

47 Kritisch mit der Forderung nach weitergehenden Begrenzungen Jescheck/Weigend, Strafrecht AT, 5. Aufl.1996, S. 87, § 9 II 2, S. 814, § 77 V 1.

48 Siehe z.B. Schmidt-Aßmann in Maunz/Dürig, Grundgesetz, Kommentar, 1958 ff (Dezember 1992), Art. 103 Abs. 3 Rn. 266, 278, 279, 288, 289; Rüping, Kommentar zum Bonner Grundgesetz, 1950 ff(Oktober 1982), Art. 103 Abs. 3 Rn. 47 unter Hinweis auf BayVerfGH 12, 168, $169 \mathrm{f}$.

49 Jescheck/Weigend, a.a.O., S. 804, § 77 I 5. Siehe im Einzelnen dazu den „Fall Stein“, BVerfGE 70, $297 \mathrm{ff}$. 
Dies ist jedoch eine Relativierung grundgesetzlicher Festlegungen, die in diesem Grundrechtsartikel an sich nicht angelegt ist. ${ }^{50}$

Historisch ist der Grundsatz ne bis in idem ein striktes, vorbehaltloses, nicht auf Abwägung bedachtes Verbot der Mehrfachbestrafung. Art. 103 Abs. 3 GG ist zwar ein verbal anerkannter, hochrangiger, fundamentaler Grundsatz eines rechtsstaatlichen Strafrechts, zugleich aber wirkungslos beim zweispurigen System von Kriminalstrafe und Sicherungsverwahrung. Das Nebeneinander beider Institute in seiner gegenwärtigen Gestalt bedarf daher der Korrektur im Interesse einer Begrenzung der Wirkungen dieser Maßregel neben der Freiheitsstrafe.

Für die Maßregeln der Unterbringung in einem psychiatrischen Krankenhaus und in einer Entziehungsanstalt ist (in $\S 67$ Abs. 4 StGB) die Anrechnung der vorwegvollzogenen Maßregel auf die Freiheitsstrafe bis zu zwei Drittel in ihrer Höhe obligatorisch vorgesehen. Diese Anrechnung wird auch in den meisten Fällen zur Anwendung gelangen, da diese Maßregeln (nach $\S 67$ Abs. 1 StGB) vor der Freiheitsstrafe im Interesse der Besserung des Täters vollzogen werden sollen. Des Weiteren kann (nach $\S 65$ Abs. 5 StGB) der Rest der Freiheitsstrafe zur Bewährung ausgesetzt werden, wenn die vorwegvollstreckte Maßregel den Zweck der Strafe erreicht hat.

Dieses sind Auswege des Gesetzgebers, um den unerträglichen Ergebnissen zu entgehen, die durch das Kumulieren von Strafen und Maßregeln entstehen. Diese gesetzlichen Vorschriften sind Maßregel- und Strafbegrenzungen, die sich im Maßregelrecht bei gefährlichen und erziehungsbedürftigen Straftätern mit dem Ziel ihrer Besserung als notwendig erwiesen haben aufgrund des ihm zugrunde liegenden Zweckgedankens. Dementsprechend wird die obligatorisch vorgesehene Anrechnung für die Unterbringung in einem psychiatrischen Krankenhaus und in einer Entziehungsanstalt mit dem Strafzweck der Spezialprävention begründet. Diesem sei mit dem Vollzug dieser Maßregeln schon weitgehend Genüge getan.

Anders verhält es sich bei der Unterbringung in der Sicherungsverwahrung, die erst im Anschluss an die Verbüßung der Freiheitsstrafe vollstreckt wird. Für diese sieht das Gesetz keine Anrechnung der Freiheitsstrafe vor. Dieser Ausweg, um zu einer Strafbegrenzung zu gelangen, ist bei ihr auch nicht möglich, da die Sicherungsverwahrung auf unbestimmte Dauer zunächst angeordnet wird. Auch besteht für die vorwegvollzogene

50 Beispielhaft und pointiert kann die Argumentation im Einzelnen bei Roxin, Strafrecht AT, Band I, 4. Aufl. 2006, S. 96 ff., § 3 B nachgelesen werden. Roxin überwindet in seinem zweckrationalen Strafrechtssystem mit der von ihm vertretenen Straf- und Maßregelkonzeption das zweigleisige Denken und deutet beide Sanktionsarten unter Annäherung an die Einspurigkeit. Strafe und Maßregel verfolgten nämlich dasselbe Ziel, der Zweck beider Sanktionen unterscheide sich im Wesentlichen nicht. Die meisten Maßregeln wirkten nämlich auch generalpräventiv, insbesondere die Entziehung der Fahrerlaubnis und die Sicherungsverwahrung, die bei sog. Hangtätern oft mehr gefürchtet werde als die zu erwartende Strafe. Bei den Strafen und Maßregeln sei die präventive Grundtendenz dieselbe, sie würden nur in ihren rechtlichen und rechtsstaatlichen Begrenzungen voneinander abweichen. Auf das Maßregelrecht finde als begrenzendes rechtsstaatliches Korrektiv nur der Verhältnismäßigkeitsgrundsatz Anwendung. 
Freiheitsstrafe keine Möglichkeit einer Strafrestaussetzung zur Bewährung. Dieser steht die Anordnung der Sicherungsverwahrung entgegen. ${ }^{51}$

Diese Differenzierung, dass nämlich das vikariierende System von Strafe und Maßregel für die Sicherungsverwahrung nicht gilt, wird damit begründet, dass mit dieser im Unterschied zu den beiden anderen genannten Maßregeln an sich keine Besserung des Täters verfolgt werde, denn die Sicherheitsinteressen der Allgemeinheit würden bei ihr ganz im Vordergrund stehen. Die Nichtbegrenzung ist Abbild des mit der Sicherungsverwahrung verbundenen rein präventiven Zweckdenkens der vorsorglichen Gefahrenabwehr.

Aus der Perspektive der Strafzwecke ist eine Begrenzung der Sicherungsverwahrung aufgrund der vorwegvollstreckten Freiheitsstrafe jedoch begründet. In ihren Wirkungen und im praktischen Vollzug unterscheidet sich jene - wie bereits gesagt - nicht wirklich von der Freiheitsstrafe. Die Sicherungsverwahrung ist nicht nur Verwahrvollzug, sondern nach $\S 129$ S. 2 StVollzG ebenfalls am Strafzweck der Resozialisierung ausgerichtet, in welchem es heißt: "Dem Sicherungsverwahrten soll geholfen werden, sich in das Leben in Freiheit einzugliedern." Auf die Vollzugsziele der Freiheitsstrafe wird im StVollzG für die Verwahrung ausdrücklich verwiesen. ${ }^{52}$

Die grundsätzliche verfassungsrechtliche und strafrechtliche Kritik an der Sicherungsverwahrung währt schon lange. Die Forderung nach ihrer Abschaffung ${ }^{53}$ oder weitgehenden Zurückdrängung ebenso. Die folgenden bekannten verfassungsrechtlichen Einwände wurden gegen die Sicherungsverwahrung erhoben, die bereits eingehend diskutiert worden sind ${ }^{54}$ und hier nur zusammenfassend benannt werden können: ${ }^{55}$

Sie verstoße gegen die Menschenwürde des Art. 1 Abs. 1 GG, da sie lediglich dem allgemeinen Sicherungsbedürfnis diene und den Verwahrten zum Sicherungsobjekt herabwürdige und gegen das Schuldprinzip. ${ }^{56}$ Des Weiteren missachte sie das Verbot der körperlichen und seelischen Misshandlung in Art. 104 Abs. 1 S. 2 GG, ${ }^{57}$ da sie den Betroffenen wegen der langen Haftdauer und des unbestimmten Entlassungstermins besonders belaste. ${ }^{58}$ Die Sicherungsverwahrung widerspreche des Weiteren dem Gleichheitsgrundsatz, da der Verwahrte im Vollzug praktisch nicht anders behandelt werde als der zur Freiheitsstrafe Verurteilte. Gravierend ist ferner der Einwand unzureichender Bestimmtheit der materiellen Anordnungsvoraussetzungen, insb. des „Hanges zu erheblichen Straftaten“ in $\S 66$ Abs. 1 Nr. 3 StGB, ${ }^{59}$ der sich auch durch Ausle-

51 Die Aussetzung der Vollstreckung der Sicherungsverwahrung zur Bewährung durch die Strafvollstreckungskammer normiert $\S 67$ d Abs. 2 S. 1 StGB, deren Überprüfung $§ 67$ e StGB mindestens alle zwei Jahre anordnet.

52 Näheres bei Laubenthal, a.a.O., Rn. 872.

53 So. z. B. Böllinger, NK-StBG, 1. Aufl. 1995, § 61 Rn. 57.

54 Vgl. Hall, ZStW 70 (1958), S. 54; H.Mayer, Strafrecht AT, 1967, S.185, § 49 II 3; Weichert, StV 1989, S. 270 f.; Hanack, LK-StGB, 11. Aufl., § 66 Rn. 22, 22 a; Köhler, Strafrecht AT, 1997, S. 55 ff., Kap. 1/3.3 m.w.N.

55 Siehe auch Böllinger/Pollähne, NK-StGB, 2. Aufl. 2005, § 61 Rn. 24.

56 Vgl. z. B. Böllinger, NK-StGB, 1. Aufl. 1995, § 66 Rn. 34.

57 Vgl. auch Art. 3 EMRK. Vgl. zur Kritik auch Laubenthal, ZStW 116 (2004), S. 711.

58 Böllinger, NK-StGB, 1. Aufl. 1995, § 66 Rn. 39 konstatiert für den Vollzug der SV Verstöße gegen die verfassungsrechtlichen Gebote der Verhältnismäßigkeit, Gleichheit und Sozialstaatlichkeit.

59 Eingehende Kritik bei Böllinger, NK-StGB, 1. Aufl. 1995, § 66 Rn. 72 ff. m.z.N. 
gung kaum für die Praxis handhabbar präzisieren lasse und deswegen Art. 103 Abs. 2 GG missachte. ${ }^{60}$

Bei dem hier skizzierten Sachstand ist in der strafrechtlichen und verfassungsrechtlichen Diskussion der Frage der Doppelsanktionierung durch Freiheitsstrafe und anschließende Sicherungsverwahrung bis hin zur neueren Verfassungsrechtsprechung nur wenig nachgegangen worden bzw. es sind aus dieser Situation nicht zureichend praktische Folgerungen gezogen worden. Bekannt ist die bereits zitierte Formulierung vom "ungerechten Etikettenschwindel". ${ }^{61}$ Man findet sich mit dem gegenwärtigen Zustand ab. Die Sicherungsverwahrung wird trotz vielfältiger Kritik von der sog. h. M. als kriminalpolitisch notwendig anerkannt. ${ }^{62}$ Begründet wird sie mit dem Hinweis, dass im Einzelfall die Gefährlichkeit eines Täters für die Allgemeinheit so groß sein könne, dass die Schuldstrafe nicht ausreiche, um die Allgemeinheit vor seinen Angriffen in einem hinreichenden Maß zu sichern. ${ }^{63}$

\section{Zum wechselseitigen Verhältnis der Strafe und der Unterbringung in der Sicherungsverwahrung}

Die Inkonsistenz von Verfassungsrecht, Strafrecht und der Maßregel Sicherungsverwahrung besteht darin, dass diese nicht eine straftheoretisch fundierte Rechtsfolge der Tat ist, sondern die rechtswidrige Tat lediglich den Anlass bildet, eine vom Täter ausgehende Gefahr für die Allgemeinheit - nämlich die Begehung weiterer schwerer Straftaten - abzuwehren. Ihre theoretische Legitimation ist ein kaum lösbares Problem. Sie ist ein Instrument kriminalpolitischer Zweckmäßigkeit, in welcher eine Sanktionenbegrenzung gedanklich nicht angelegt ist. Sie birgt in sich die Gefahr, zur Erreichung des erwünschten Sicherungszwecks zu übermäßigen, freiheitsentziehenden Mitteln zu greifen.

Im Strafzumessungsrecht wurde durch das Doppelverwertungsverbot des $\S 46$ Abs. 3 StGB der Gefahr begegnet, ein und denselben Tatumstand wiederholt Tätern zu ihren Lasten bei der Bestimmung von Strafart und Strafmaß zuzuschreiben. Tatbestandsmerkmale dürfen bei der Festlegung der Rechtsfolge nicht nochmals in Ansatz gebracht werden, sie werden für die Strafzumessung als "verbraucht" gekennzeichnet. $\S 46$ Abs. 3 StGB ist damit Ausdruck des Grundsatzes ne bis in idem und der richterlichen Bindung an das Gesetz, d.h. an die in den Strafrechtsnormen zum Ausdruck gelangten Wertungen des Gesetzgebers.

Im Maßregelrecht hat dieser Gedanke nur indirekt und unzureichend Berücksichtigung gefunden im gesetzlich ausformulierten Verhältnismäßigkeitsgrundsatz des $\S 62$

60 Unter Hinweis auf den Doppelcharakter von Strafen und Maßregeln, welche sich tendenziell in ihrer Funktion nivellierten, sieht Böllinger, NK-StGB, 1. Aufl. 1995, § 61 Rn. 23 einen Verstoß gegen die Grundsätze „nulla poena sine lege“, „,ne bis in idem“ und das Bestimmtheitsgebot.

61 Naucke, Strafrecht, 10. Aufl. 2002, S. 99, § 3 Rn. 34; ebenso Böllinger/Pollähne, NK-StGB, 2. Aufl. 2005, § 61 Rn. 21 „Quasi-Strafen“.

62 Vgl. z.B. Meier, Strafrechtliche Sanktionen, 3. Aufl., 2009, S. 297, Teil 5 3.3.1.

63 Vgl. z. B. Jescheck/Weigend, a.a.o., S. 86, § 9 II 1, S. 802 f., § 77 I 1 jeweils m.w.N. 
StGB i. S. e. Übermaßverbotes. ${ }^{64}$ Nach ihm darf die Maßregel insbesondere zur Bedeutung der vom Täter begangenen und zu erwartenden Taten nicht außer Verhältnis stehen. Es fehlt bei der Sicherungsverwahrung zwecks Vermeidung einer wiederholten Verwertung von Merkmalen der Straftat und ihrer Rechtsfolgenzumessung eine Regelung entsprechend der des $\S 46$ Abs. 3 StGB.

Umstände, die bereits bei der Bestimmung der Höhe der Freiheitsstrafe in der Strafzumessung berücksichtigt worden sind, dürften nicht nochmals bei der Subsumtion der Voraussetzungen der Sicherungsverwahrung in Ansatz gebracht werden. Solche zu Lasten des Delinquenten bereits bewerteten Umstände sind strafjuristisch „verbraucht“.

Oder aber: Bei der Strafzumessungsentscheidung nach § 46 StGB dürfen, wenn die Anordnung einer Sicherungsverwahrung neben einer Freiheitsstrafe erfolgen soll, nicht bereits Umstände straferhöhend berücksichtigt werden, mit Hilfe derer die Maßregel anschließend begründet werden soll. Das Gericht wäre also ggf. gehalten, eine Freiheitsstrafe von geringerer Höhe zu verhängen, damit die Tatumstände bzw. die Merkmale in der Person des Täters für die Begründung der Sicherungsverwahrung noch zur Verfügung stehen.

Deutlich wird hieran das gegenseitige Abhängigkeitsverhältnis, in welchem die Strafen und die Maßregeln zueinander stehen. ${ }^{65}$ Bei dem derzeitigen Nebeneinander dieser Rechtsfolgen und den Voraussetzungen ihrer Anordnung wird diese Abhängigkeit übergangen. ${ }^{66}$

Unklar ist jedoch in der Rechtsprechung und in der Literatur, ob und wie weit bei der Strafzumessung die Wirkung einer gleichzeitig verhängten Maßregel berücksichtigt werden darf. So hält es der $\mathrm{BGH}^{67}$ nicht für möglich, allein im Hinblick auf eine gleichzeitig verhängte Maßregel das Maß der schuldangemessenen Strafe zu unterschreiten. Andererseits ist der Richter nicht befugt, an Stelle der Sicherungsverwahrung eine län-

64 Der Verhältnismäßigkeitsgrundsatz übernimmt bei den Maßregeln die begrenzende Funktion, welche das Schuldprinzip bei den Strafen in wesentlich ausgeprägter Weise übernimmt, vgl. Jescheck/Weigend, a.a.O., S. 85, § 9 I 2. Ob er dieses zu leisten vermag, wird hier aber gerade in Frage gestellt.

65 Ebenso Naucke, Strafrecht, 10. Aufl. 2002, S. 105, § 3 Rn. 53 f. mit Hinweis auf in der Strafrechtsgeschichte belegte Entwicklungslinien.

66 Böllinger/Pollähne, NK-StGB, 2. Aufl. 2005, § 66 Rn. 5 beschreibt das Nebeneinander von Strafe und Maßregel abweichend hiervon als „funktional äquivalente Regelungen“, welches einer Gleichsetzung gleichkommt, was jedoch in der Sache nicht der Fall ist. Übersehen wird, dass die SV den Betroffenen ungleich härter trifft als die Strafe. Vgl. hierzu im einzelnen Sander, Grenzen instrumenteller Vernunft im Strafrecht, 2007, S. 268, die darauf hinweist, dass (lediglich) nach präventiver Systemlogik äquivalente Sanktionsformen vorliegen. Rissing van Scan, LK-StGB, 12. Aufl., §66 Rn. 23 beklagt die Einebnung der Unterschiede zwischen schuldgebundener Strafe und schuldunabhängiger SV durch die Reformgesetzgebung, welche ein Widerspruch zum Konzept einer präventiven Maßregel sei, die erst dann anzuwenden sei, wenn es zu einer Straftat gekommen sei. Auch sei nach Einführung der $\S \S 66 \mathrm{a}, \mathrm{b}$ StGB von einer strafmildernden Berücksichtigung der Anordnung der SV auf die Strafe kaum auszugehen bzw. diese nicht mehr geboten, da anderenfalls ohne sachlichen Grund eine Besserstellung derjenigen Straftäter erfolgen würde, die bereits anfänglich zu SV verurteilt worden seien. Zutreffend wird von einer Wechselwirkung zwischen Maßregelverhängung und Strafzumessung ausgegangen.

67 BGHSt 24, 132. 
gere Freiheitsstrafe zu verhängen. ${ }^{68}$ In der Rechtsprechung ist oft anerkannt worden, dass das Strafmaß durch die gleichzeitige Anordnung einer Maßregel „beeinflusst“ werden kann, die Strafe - bei alleiniger Verhängung - also strenger ausgefallen wäre. Der $\mathrm{BGH}^{69}$ betont die Möglichkeit und Notwendigkeit Strafen und Maßregeln ,aufeinander abzustimmen“. Die durch die Anschlussvollstreckung der Maßregel entstehende Zusatzbelastung des Angeklagten müsse bei der Strafzumessung zu seinen Gunsten berücksichtigt werden, weil sich dadurch insgesamt die Freiheitsentziehung verlängere. ${ }^{70}$ Vorhandene Ansätze in der Literatur versuchen diese Problematik im Rahmen der Strafzumessungsgrundsätze des $\S 46$ StGB zu bewältigen.

Durch das Nebeneinander von Strafe und Sicherungsverwahrung leidet das Rechtsfolgensystem des Weiteren unter normativen Ungereimtheiten seitens der Gesetzgebung. Die Sicherungsverwahrung setzt niedrigere Strafrahmen und damit leichtere Freiheitsstrafen voraus, als ein Rechtssystem ohne dieses Institut, damit der mit ihr verbundene Zweck der Sicherung der Allgemeinheit vor gefährlichen Wiederholungstätern praktisch relevant werden kann. Hohe Strafrahmen auf deren Grundlage vergleichsweise harte Freiheitsstrafen verhängt werden können, enthalten bereits das Sicherungsinteresse der Gemeinschaft vor der erneuten Begehung von Straftaten durch den Verurteilten. Die Notwendigkeit einer zusätzlichen Sicherungsverwahrung tritt dann zurück. Die gesetzlichen Anforderungen für ihre Anordnung müssten dementsprechend höher sein. Dies ist wieder Abbild der wechselseitigen Abhängigkeit von Strafe und Maßregel.

Die Wertungen des Gesetzgebers entsprechen nicht dieser gegenseitigen Abhängigkeit. Die Erhöhung von Strafrahmen müsste zur Konsequenz haben, dass strengere Anforderungen an die Anordnungsvoraussetzungen der Sicherungsverwahrung zu stellen sind. Dies ist aber beispielsweise anlässlich des 6 . Strafrechtsreformgesetzes mit seinen erhöhten Strafrahmen gerade nicht geschehen, sondern höhere Strafen gingen einher mit einem strengeren Maßregelrecht. Daher wird in der strafrechtlichen Diskussion nicht nur von einer Renaissance der Sicherungsverwahrung, sondern auch von einer Renaissance der Freiheitstrafe ${ }^{71}$ gesprochen. Diese Renaissance im Rechtsfolgenrecht bedarf dringend einer grundlegenden Revision.

68 BGH bei Dallinger, MDR 1973, S. 727.

69 MDR 1985, S. 282.

70 BGH, MDR 1985, S. 90.

71 Schneider, Festschrift für Seebode, 2008, S. 525 ff.; Schäfer/Sander/van Gemmeren, Praxis der Strafzumessung, 4. Aufl. 2008, S. 5 Rn. 12. 